\title{
The Multisoliton Solutions for the $(2+1)$-Dimensional Sawada-Kotera Equation
}

\author{
Zhenhui Xu, ${ }^{1}$ Hanlin Chen, ${ }^{2}$ and Wei Chen ${ }^{3}$ \\ ${ }^{1}$ Applied Technology College, Southwest University of Science and Technology, Mianyang 621010, China \\ ${ }^{2}$ School of Science, Southwest University of Science and Technology, Mianyang 621010, China \\ ${ }^{3}$ School of Mathematics and Computer Science, Mianyang Normal University, Mianyang 621000, China
}

Correspondence should be addressed to Zhenhui Xu; xuzhenhui19@163.com

Received 13 November 2012; Revised 19 December 2012; Accepted 1 January 2013

Academic Editor: Lan Xu

Copyright (C) 2013 Zhenhui Xu et al. This is an open access article distributed under the Creative Commons Attribution License, which permits unrestricted use, distribution, and reproduction in any medium, provided the original work is properly cited.

Applying bilinear form and extended three-wavetype of ansätz approach on the $(2+1)$-dimensional Sawada-Kotera equation, we obtain new multisoliton solutions, including the double periodic-type three-wave solutions, the breather two-soliton solutions, the double breather soliton solutions, and the three-solitary solutions. These results show that the high-dimensional nonlinear evolution equation has rich dynamical behavior.

\section{Introduction}

As is well known that the exact solutions of nonlinear evolution equations play an important role in nonlinear science field, especially in nonlinear physical science since they can provide much physical information and more insight into the physical aspects of the problem and thus lead to further applications. The search for exact solutions of nonlinear partial differential equations has long been an interesting and hot topic in nonlinear mathematical physics. Consequently, many methods are available to look for exact solutions of nonlinear evolution equations, such as the inverse scattering method, the Lie group method, the mapping method, Exp-function method, and ansätz technique [1-4]. Very recently, Wang et al. [5] proposed a new technique called extended threewave approach to seek multiwave solutions for integrable equations, and this method has been used to investigate several equations $[6,7]$. In this paper, we consider the following Sawada-Kotera equation:

$$
\begin{aligned}
u_{t}= & \left(u_{x x x x}+5 u u_{x x}+\frac{5}{3} u^{3}+5 u_{x y}\right)_{x} \\
& -5 \int\left(u_{y y}\right) d x+5 u u_{y}+5 u_{x} \int\left(u_{y}\right) d x .
\end{aligned}
$$

Equation (1) was derived by B. G. Konopelchenko and V. G. Dubrovsky, and was called the Sawada-Kotera (SK) equation; for example, see [8]. By means of the two-soliton method, the exact periodic soliton solutions, $\mathrm{N}$-soliton solutions, and traveling wave solutions of the SK equation were found [8$10]$.

In this paper, we discuss further the $(2+1)$-dimensional SK equation, by using bilinear form and extended three-wave type of ansätz approach, respectively [5,11-15], and some new multisoliton solutions are obtained.

\section{The Multisoliton Solutions}

We assume

$$
u=-2(\ln f)_{x x},
$$

where $f=f(x, y, t)$ is an unknown real function. Substituting (2) into (1), we can reduce (1) into the following equation [8]:

$$
\left(D_{x}^{6}+5 D_{y} D_{x}^{3}-5 D_{y}^{2}+D_{x} D_{t}\right) f \cdot f=0,
$$


where the Hirota bilinear operator $D$ is defined by $(n, m \geq 0)$

$$
\begin{array}{rl}
D_{x}^{m} D_{t}^{n} & f(x, t) \cdot g(x, t) \\
= & \left(\frac{\partial}{\partial x}-\frac{\partial}{\partial x^{\prime}}\right)^{m}\left(\frac{\partial}{\partial t}-\frac{\partial}{\partial t^{\prime}}\right)^{n} \\
& \times\left.\left[f(x, t) g\left(x^{\prime}, t^{\prime}\right)\right]\right|_{x^{\prime}=x, t^{\prime}=t^{\prime}}
\end{array}
$$

Now we suppose the solution of (3) as

$$
f=e^{-\xi}+\delta_{1} \cos (\eta)+\delta_{2} \cosh (\gamma)+\delta_{3} e^{\xi}
$$

where $\xi=a_{1} x+b_{1} y+c_{1} t, \eta=a_{2} x+b_{2} y+c_{2} t, \gamma=a_{3} x+$ $b_{3} y+c_{3} t$, and $a_{i}, b_{i}$, and $c_{i}(i=1,2,3)$ are some constants to be determined later. Substituting (5) into (3) and equating all the coefficients of different powers of $e^{\xi}, e^{-\xi}, \sin (\eta), \cos (\eta)$, $\sinh (\gamma), \cosh (\gamma)$, and the constant term to zero, we can obtain a set of algebraic equations for $a_{i}, b_{i}, c_{i}$, and $\delta_{j}(i=1,2,3$; $j=1,2,3)$. Solving the system with the aid of Maple, we get the following results.

Case 1. If $a_{2}=0$, then

$$
\begin{gathered}
b_{1}=-\frac{1}{4} a_{1}\left(4 a_{1}^{2}+3 a_{3}^{2}\right), \quad b_{2}=\frac{3}{2} i a_{1}^{2} a_{3}, \\
b_{3}=-\frac{1}{4} a_{3}\left(6 a_{1}^{2}+a_{3}^{2}\right), \quad \delta_{2}=-\frac{\delta_{1} a_{1}^{2}}{a_{1}^{2}-a_{3}^{2}}, \\
\delta_{3}=\delta_{3}, \\
c_{1}=\frac{9}{16} a_{1}\left(5 a_{3}^{4}+40 a_{1}^{2} a_{3}^{2}+16 a_{1}^{4}\right), \\
c_{2}=-\frac{45}{4} i a_{1}^{2} a_{3}\left(2 a_{1}^{2}+a_{3}^{2}\right), \\
c_{3}=\frac{9}{16} a_{3}\left(a_{3}^{4}+20 a_{1}^{2} a_{3}^{2}+40 a_{1}^{4}\right),
\end{gathered}
$$

where $a_{1}, a_{3}, \delta_{1}$, and $\delta_{3}$ are free real constants. Substituting (6) into (5) and taking $\delta_{3}>0$, we have

$$
\begin{aligned}
f_{1}= & 2 \sqrt{\delta_{3}} \cosh \left(a_{1} x+K_{1} y+L_{1} t+\frac{1}{2} \ln \left(\delta_{3}\right)\right) \\
& -\delta_{1} \cosh \left(M_{1} y+N_{1} t\right)-\frac{\delta_{1} a_{1}^{2}}{a_{1}^{2}-a_{3}^{2}} \\
& \times \cosh \left(a_{3} x-H_{1} y+J_{1} t\right),
\end{aligned}
$$

where $K_{1}=(1 / 4) a_{1}\left(4 a_{1}^{2}+3 a_{3}^{2}\right), L_{1}=(9 / 16) a_{1}\left(5 a_{3}^{4}+40 a_{1}^{2} a_{3}^{2}+\right.$ $\left.16 a_{1}^{4}\right), M_{1}=-(3 / 2) a_{1}^{2} a_{3}, N_{1}=(45 / 4) a_{1}^{2} a_{3}\left(2 a_{1}^{2}+a_{3}^{2}\right), H_{1}=$ $(1 / 4) a_{3}\left(6 a_{1}^{2}+a_{3}^{2}\right)$, and $J_{1}=(9 / 16) a_{3}\left(a_{3}^{4}+20 a_{1}^{2} a_{3}^{2}+40 a_{1}^{4}\right)$.
Substituting (7) into (2) yields the three-soliton solution of SK equation as follows:

$$
\begin{gathered}
u_{1}=-\left(2 \left[2 \sqrt{\delta_{3}} a_{1}^{2} \cosh \left(\xi_{1}+\frac{1}{2} \ln \left(\delta_{3}\right)\right)\right.\right. \\
\left.\left.-\frac{\delta_{1} a_{1}^{2} a_{3}^{2} \cosh \left(\eta_{1}\right)}{a_{1}^{2}-a_{3}^{2}}\right]\right) \\
\times\left(2 \sqrt{\delta_{3}} \cosh \left(\xi_{1}+\frac{1}{2} \ln \left(\delta_{3}\right)\right)\right. \\
+\left[\left(2 \left(2 \sqrt{\delta_{3}} a_{1} \sinh \left(\xi_{1}+\frac{1}{2} \ln \left(\delta_{3}\right)\right)\right.\right.\right. \\
\left.-\frac{\delta_{1} a_{1}^{2} \cosh \left(\eta_{1}\right)}{a_{1}^{2}-a_{3}^{2}}-\delta_{1} \cosh \left(\gamma_{1}\right)\right)^{-1} \\
\left.\left.\quad-\frac{\delta_{1} a_{1}^{2} a_{3} \sinh \left(\eta_{1}\right)}{\left(a_{1}^{2}-a_{3}^{2}\right)}\right)\right) \\
\times\left(2 \sqrt{\delta_{3}} \cosh \left(\xi_{1}+\frac{1}{2} \ln \left(\delta_{3}\right)\right)\right. \\
\left.\left.\quad-\frac{\delta_{1} a_{1}^{2} \cosh \left(\eta_{1}\right)}{a_{1}^{2}-a_{3}^{2}}-\delta_{1} \cosh \left(\gamma_{1}\right)\right)^{-1}\right]^{2}
\end{gathered}
$$

where $\xi_{1}=a_{1} x+K_{1} y+L_{1} t, \eta_{1}=a_{3} x-H_{1} y+J_{1} t$, and $\gamma_{1}=M_{1} y+N_{1} t$.

If taking $a_{3}=i A_{3}$ in (7), then we have

$$
\begin{aligned}
f_{2}= & 2 \sqrt{\delta_{3}} \cosh \left(a_{1} x+K_{2} y+L_{2} t+\frac{1}{2} \ln \left(\delta_{3}\right)\right) \\
& +\delta_{1} \cos \left(M_{2} y+N_{2} t\right) \\
& -\frac{\delta_{1} a_{1}^{2} \cos \left(A_{3} x-H_{2} y+J_{2} t\right)}{a_{1}^{2}+A_{3}^{2}},
\end{aligned}
$$

where $\delta_{3}>0, K_{2}=-(1 / 4) a_{1}\left(4 a_{1}^{2}-3 A_{3}^{2}\right), L_{2}=(9 / 16) a_{1}\left(5 A_{3}^{4}-\right.$ $\left.40 a_{1}^{2} A_{3}^{2}+16 a_{1}^{4}\right), M_{2}=(3 / 2) a_{1}^{2} A_{3}, N_{2}=-(45 / 4) a_{1}^{2} A_{3}\left(2 a_{1}^{2}-\right.$ $\left.A_{3}^{2}\right), H_{2}=A_{3} x-(1 / 4) A_{3}\left(6 a_{1}^{2}-A_{3}^{2}\right)$, and $J_{2}=(9 / 16) A_{3}\left(A_{3}^{4}-\right.$ $\left.20 a_{1}^{2} A_{3}^{2}+40 a_{1}^{4}\right)$. Substituting (9) into (2) yields the double breather soliton solution of SK equation as follows:

$$
\begin{gathered}
u_{2}=-\left(2 \left[2 a_{1}^{2} \sqrt{\delta_{3}} \cosh \left(\xi_{2}+\frac{1}{2} \ln \left(\delta_{3}\right)\right)\right.\right. \\
\left.\left.+\frac{\delta_{1} a_{1}^{2} A_{3}^{2} \cos \left(\eta_{2}\right)}{a_{1}^{2}+A_{3}^{2}}\right]\right) \\
\times\left(2 \sqrt{\delta_{3}} \cosh \left(\xi_{2}+\frac{1}{2} \ln \left(\delta_{3}\right)\right)\right. \\
\left.+\delta_{1} \cos \left(\gamma_{2}\right)-\frac{\delta_{1} a_{1}^{2} \cos \left(\eta_{2}\right)}{a_{1}^{2}+A_{3}^{2}}\right)^{-1}
\end{gathered}
$$




$$
\begin{gathered}
+2\left[\left(2 a_{1} \sqrt{\delta_{3}} \sinh \left(\xi_{2}+\frac{1}{2} \ln \left(\delta_{3}\right)\right)\right.\right. \\
\left.+\frac{\delta_{1} a_{1}^{2} A_{3} \sin \left(\eta_{2}\right)}{a_{1}^{2}+A_{3}^{2}}\right) \\
\times\left(2 \sqrt{\delta_{3}} \cosh \left(\xi_{2}+\frac{1}{2} \ln \left(\delta_{3}\right)\right)\right. \\
\left.\left.+\delta_{1} \cos \left(\gamma_{2}\right)-\frac{\delta_{1} a_{1}^{2} \cos \left(\eta_{2}\right)}{a_{1}^{2}+A_{3}^{2}}\right)^{-1}\right]^{2}
\end{gathered}
$$

where $\xi_{2}=a_{1} x+K_{2} y+L_{2} t, \eta_{2}=A_{3} x-H_{2} y+J_{2} t$, and $\gamma_{2}=M_{2} y+N_{2} t$.

Case 2. If $a_{2} \neq 0$, then

$$
\begin{aligned}
& b_{1}=-a_{1}^{3}, \quad b_{2}=a_{2}^{3}, \quad b_{3}=-a_{3}^{3}, \\
& \delta_{1}=\delta_{1}, \quad \delta_{2}=\delta_{2}, \quad \delta_{3}=\delta_{3}, \\
& c_{1}=9 a_{1}^{5}, \quad c_{2}=9 a_{2}^{5}, \quad c_{3}=9 a_{3}^{5},
\end{aligned}
$$

where $a_{1}, a_{2}, a_{3}, \delta_{1}, \delta_{2}$, and $\delta_{3}$ are free real constants. Substituting (11) into (5) and taking $\delta_{3}>0$, we have

$$
\begin{aligned}
f_{3}= & 2 \sqrt{\delta_{3}} \cosh \left(a_{1} x-a_{1}^{3} y+9 a_{1}^{5} t+\frac{1}{2} \ln \left(\delta_{3}\right)\right) \\
& +\delta_{1} \cos \left(a_{2} x+a_{2}^{3} y+9 a_{2}^{5} t\right) \\
& +\delta_{2} \cosh \left(a_{3} x-a_{3}^{3} y+9 a_{3}^{5} t\right) .
\end{aligned}
$$

Substituting (12) into (2) yields the breather two-soliton solution of SK equation as follows:

$$
\begin{aligned}
u_{3}= & -\left(2 \left[2 \sqrt{\delta_{3}} a_{1}^{2} \cosh \left(\xi_{3}+\frac{1}{2} \ln \left(\delta_{3}\right)\right)\right.\right. \\
& \left.\left.-\delta_{1} a_{2}^{2} \cos \left(\eta_{3}\right)+\delta_{2} a_{3}^{2} \cosh \left(\gamma_{3}\right)\right]\right) \\
\times & \left(2 \sqrt{\delta_{3}} \cosh \left(\xi_{3}+\frac{1}{2} \ln \left(\delta_{3}\right)\right)\right. \\
+ & \left.\delta_{1} \cos \left(\eta_{3}\right)+\delta_{2} \cosh \left(\gamma_{3}\right)\right)^{-1} \\
+ & \left(2 \left(2 \sqrt{\delta_{3}} a_{1} \sinh \left(\xi_{3}+\frac{1}{2} \ln \left(\delta_{3}\right)\right)\right.\right. \\
& \left.\left.\quad-\delta_{1} a_{2} \sin \left(\eta_{3}\right)+\delta_{2} a_{3} \sinh \left(\gamma_{3}\right)\right)\right) \\
& \quad\left(2 \sqrt{\delta_{3}} \cosh \left(\xi_{3}+\frac{1}{2} \ln \left(\delta_{3}\right)\right)\right. \\
& \left.\left.+\delta_{1} \cos \left(\eta_{3}\right)+\delta_{2} \cosh \left(\gamma_{3}\right)\right)^{-1}\right]^{2}
\end{aligned}
$$

where $\xi_{3}=a_{1} x-a_{1}^{3} y+9 a_{1}^{5} t, \eta_{3}=a_{2} x+a_{2}^{3} y+9 a_{2}^{5} t$, and $\gamma_{3}=a_{3} x-a_{3}^{3} y+9 a_{3}^{5} t$.
The expression $\left(u_{3}\right)$ is the breather two-soliton solution of SK equation which is a periodic wave in $x, y$ and meanwhile is a two-soliton in $x, y$ (refer to Figure $1(\mathrm{~b})$ ).

Case 3. If $a_{2}=b_{1}=0$, then

$$
\begin{gathered}
a_{1}=2 a_{3}, \quad b_{2}=\sqrt{21} a_{3}^{3}, \quad b_{3}=-\frac{3}{2} a_{3}^{3}, \\
c_{1}=-\frac{169}{2} a_{3}^{5}, \\
c_{2}=-20 \sqrt{21} a_{3}^{5}, \quad c_{3}=-\frac{349}{4} a_{3}^{5}, \\
\delta_{3}=\frac{5}{152} \delta_{2}^{2}-\frac{7}{228} \delta_{1}^{2},
\end{gathered}
$$

where $a_{3}, \delta_{1}$, and $\delta_{2}$ are free real constants. Substituting (14) into (5) and taking $\delta_{3}>0$, we have

$$
\begin{aligned}
f_{4}= & 2 \sqrt{\frac{5}{152} \delta_{2}^{2}-\frac{7}{228} \delta_{1}^{2}} \\
& \times \cosh \left(-2 a_{3} x+\frac{169}{2} a_{3}^{5} t-\frac{1}{2} \ln \left(\frac{5}{152} \delta_{2}^{2}-\frac{7}{228} \delta_{1}^{2}\right)\right) \\
& +\delta_{1} \cos \left(-\sqrt{21} a_{3}^{3} y+20 \sqrt{21} a_{3}^{5} t\right) \\
& +\delta_{2} \cosh \left(-a_{3} x+\frac{3}{2} a_{3}{ }^{3} y+\frac{349}{4} a_{3}^{5} t\right),
\end{aligned}
$$

where $(5 / 152) \delta_{2}^{2}-(7 / 228) \delta_{1}^{2}>0$. Substituting (15) into (2) yields the breather two-soliton solution of SK equation as follows:

$$
\begin{aligned}
& u_{4}=\left(2 \left[8 \sqrt{K_{4}} a_{3}^{2} \cosh \left(\xi_{4}-\frac{1}{2} \ln \left(K_{4}\right)\right)\right.\right. \\
&\left.\left.+\delta_{2} a_{3}^{2} \cosh \left(\eta_{4}\right)\right]\right) \\
& \times\left(2 \sqrt{K_{4}} \cosh \left(\xi_{4}-\frac{1}{2} \ln \left(K_{4}\right)\right)\right. \\
&+\left.\delta_{1} \cos \left(\gamma_{4}\right)+\delta_{2} \cosh \left(\eta_{4}\right)\right)^{-1} \\
&+2\left[\left(4 \sqrt{K_{4}} a_{3} \sinh \left(\xi_{4}-\frac{1}{2} \ln \left(K_{4}\right)\right)\right.\right. \\
&\left.+\delta_{2} a_{3} \sinh \left(\eta_{4}\right)\right) \\
& \times\left(2 \sqrt{K_{4}} \cosh \left(\xi_{4}-\frac{1}{2} \ln \left(K_{4}\right)\right)\right. \\
&\left.\left.+\delta_{1} \cos \left(\gamma_{4}\right)+\delta_{2} \cosh \left(\eta_{4}\right)\right)^{-1}\right]^{2}
\end{aligned}
$$

where $K_{4}=(5 / 152) \delta_{2}^{2}-(7 / 228) \delta_{1}^{2}, \xi_{4}=-2 a_{3} x+(169 / 2) a_{3}^{5} t$, $\eta_{4}=-a_{3} x+(3 / 2) a_{3}^{3} y+(349 / 4) a_{3}^{5} t$, and $\gamma_{4}=-\sqrt{21} a_{3}^{3} y+$ $20 \sqrt{21} a_{3}^{5} t$. 


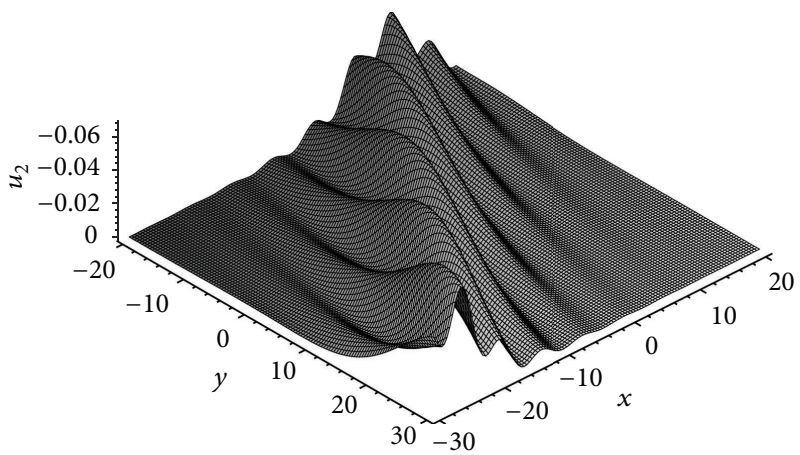

(a)

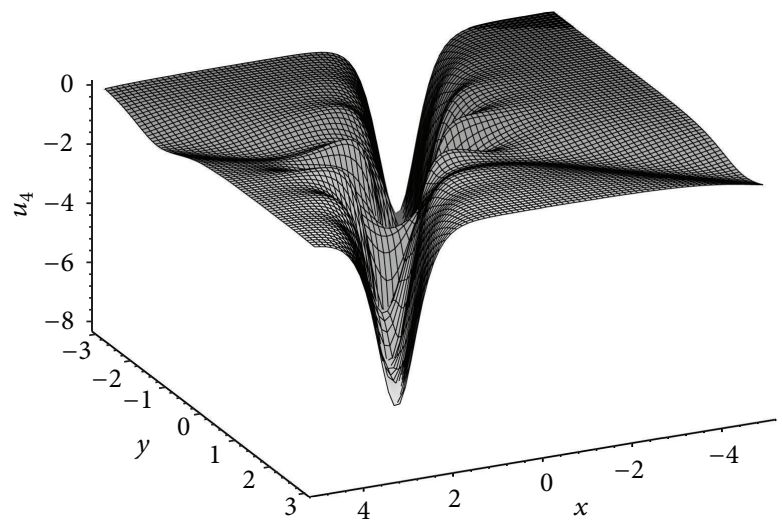

(c)

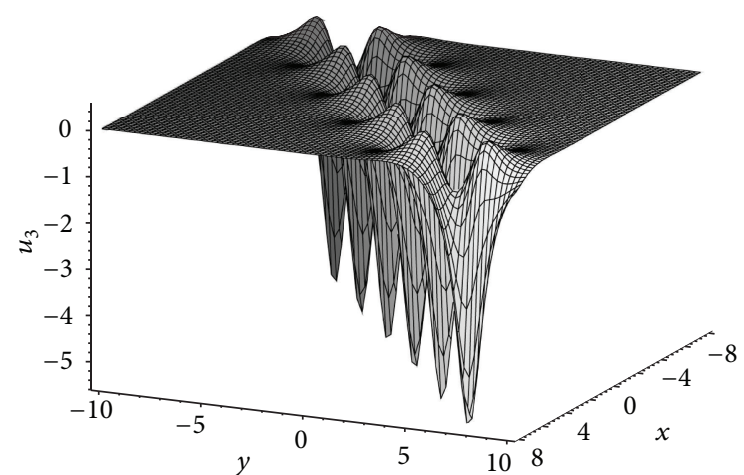

(b)

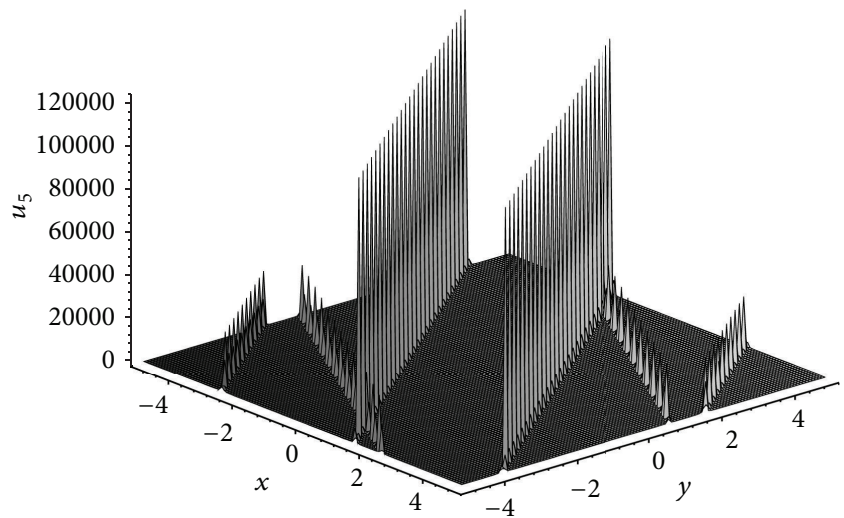

(d)

Figure 1: (a) The figure of $u_{2}$ as $\delta_{1}=1, \delta_{3}=1$, and $t=1$. (b) The figure of $u_{3}$ as $\delta_{1}=\sqrt{2}, \delta_{2}=1$, and $t=0$. (c) The figure of $u_{4}$ as $\delta_{1}=\sqrt{2}$, $\delta_{2}=\sqrt{5}$, and $t=0.005$. (d) The figure of $u_{5}$ as $\delta_{1}=1, \delta_{2}=1$, and $t=0$.

The expression $\left(u_{4}\right)$ is the breather two-soliton solution of SK equation which is a periodic wave in $y$ - $t$ and meanwhile is a two-soliton in $x, y$ and in $x$-t, respectively (refer to Figure 1(c)).

Notice that $u_{3}$ and $u_{4}$ are also the breather two-soliton solutions, but their structure is different, because the two wave propagation directions are different in the $u_{3}$ and $u_{4}$, respectively (refer to Figures $1(\mathrm{~b})$ and $1(\mathrm{c})$ ).

If taking $a_{1}=i A_{1}, a_{3}=i A_{3}$ in (12), then we have

$$
\begin{aligned}
f_{5}= & 2 \cos \left(A_{1} x+A_{1}^{3} y+9 A_{1}^{5} t\right) \\
& +\delta_{1} \cos \left(a_{2} x+a_{2}^{3} y+9 a_{2}^{5} t\right) \\
& +\delta_{2} \cos \left(A_{3} x+A_{3}^{3} y+9 A_{3}^{5} t\right),
\end{aligned}
$$

when $\delta_{3}=1$. Substituting (17) into (2) gives the doubleperiodic three-wave solution of SK equation as follows:

$$
\begin{aligned}
u_{5}= & \frac{2\left[2 A_{1}^{2} \cos \left(\xi_{5}\right)+\delta_{1} a_{2}^{2} \cos \left(\eta_{5}\right)+\delta_{2} A_{3}^{2} \cos \left(\gamma_{5}\right)\right]}{2 \cos \left(\xi_{5}\right)+\delta_{1} \cos \left(\eta_{5}\right)+\delta_{2} \cos \left(\gamma_{5}\right)} \\
& +2\left[\frac{2 A_{1} \sin \left(\xi_{5}\right)+\delta_{1} a_{2} \sin \left(\eta_{5}\right)+\delta_{2} A_{3} \sin \left(\gamma_{5}\right)}{2 \cos \left(\xi_{5}\right)+\delta_{1} \cos \left(\eta_{5}\right)+\delta_{2} \cos \left(\gamma_{5}\right)}\right]^{2},
\end{aligned}
$$

where $\xi_{5}=A_{1} x+A_{1}^{3} y+9 A_{1}^{5} t, \eta_{5}=a_{2} x+a_{2}^{3} y+9 a_{2}^{5} t$, and $\gamma_{5}=A_{3} x+A_{3}^{3} y+9 A_{3}^{5} t$.

\section{Conclusion}

By using bilinear form and extended three-wave type of ansätz approach, we discuss further the $(2+1)$-dimensional 
Sawada-Kotera equation and find some new multisoliton solutions. The result shows that the extended three-wave type of ansätz approach may provide us with a straightforward and effective mathematical tool for seeking multiwave solutions of high-dimensional nonlinear evolution equations.

\section{Acknowledgments}

This work was supported by Chinese Natural Science Foundation Grant nos. 11061028, 10971169. Sichuan Educational Science Foundation Grant no. 09zc008.

\section{References}

[1] M. J. Abolowitz and P. A. Clarkson, Solitons, Nonlinear Evolution Equations and Inverse Scattering Transform, Cambridge University Press, Cambridge, UK, 1991.

[2] A. Maccari, "Chaos, solitons and fractals in the nonlinear Dirac equation," Physics Letters A, vol. 336, no. 2-3, pp. 117-125, 2005.

[3] Z. Feng, "Comment on "On the extended applications of homogeneous balance method'"' Applied Mathematics and Computation, vol. 158, no. 2, pp. 593-596, 2004.

[4] X. Q. Liu, H. L. Chen, and Y. Q. Lü, "Explicit solutions of the generalized KdV equations with higher order nonlinearity," Applied Mathematics and Computation, vol. 171, no. 1, pp. 315319, 2005 .

[5] C. Wang, Z. Dai, and L. Liang, "Exact three-wave solution for higher dimensional KdV-type equation," Applied Mathematics and Computation, vol. 216, no. 2, pp. 501-505, 2010.

[6] Z. Dai, S. Lin, H. Fu, and X. Zeng, "Exact three-wave solutions for the KP equation," Applied Mathematics and Computation, vol. 216, no. 5, pp. 1599-1604, 2010.

[7] Z. Li and Z. Dai, "Exact periodic cross-kink wave solutions and breather type of two-solitary wave solutions for the $(3+1)$ dimensional potential-YTSF equation," Computers and Mathematics with Applications, vol. 61, no. 8, pp. 1939-1945, 2011.

[8] H. Y. Ruan, "Interactions between two-periodic solitons in the $(2+1)$-dimensional Sawada-Kotera equations," Acta Physica Sinica, vol. 53, no. 6, pp. 1618-1622, 2004.

[9] H. M. Fu and Z. D. Dai, "Periodic soliton wave solutions for twodimension S-K equation," Journal of Xuzhou Normal University, vol. 27, no. 4, pp. 20-22, 2009.

[10] Z. Y. Wang and H. L. Lv, "Travelling wave solutions of $(2+$ 1)-dimensional Sawada-Kotera equation," Journal of Liaocheng University, vol. 23, no. 4, 2010.

[11] W. X. Ma and E. Fan, "Linear superposition principle applying to Hirota bilinear equations," Computers and Mathematics with Applications, vol. 61, no. 4, pp. 950-959, 2011.

[12] Z. Dai, J. Huang, M. Jiang, and S. Wang, "Homoclinic orbits and periodic solitons for Boussinesq equation with even constraint," Chaos, Solitons and Fractals, vol. 26, no. 4, pp. 1189-1194, 2005.

[13] Z. H. Xu, D. Q. Xian, and H. L. Chen, "New periodic solitarywave solutions for the Benjiamin Ono equation," Applied Mathematics and Computation, vol. 215, no. 12, pp. 4439-4442, 2010.

[14] Z. Dai, S. Li, Q. Dai, and J. Huang, "Singular periodic soliton solutions and resonance for the Kadomtsev-Petviashvili equation," Chaos, Solitons and Fractals, vol. 34, no. 4, pp. 1148-1153, 2007.
[15] J. H. He, "Asymptotic methods for solitary solutions and compactons," Abstract and Applied Analysis, vol. 2012, Article ID 916793, 130 pages, 2012. 


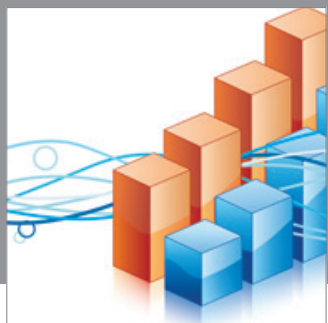

Advances in

Operations Research

mansans

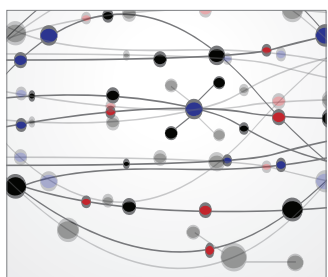

The Scientific World Journal
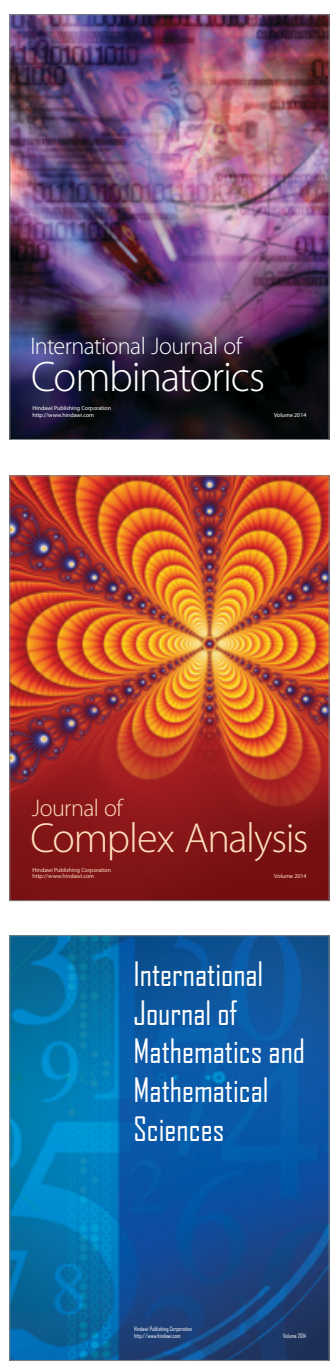
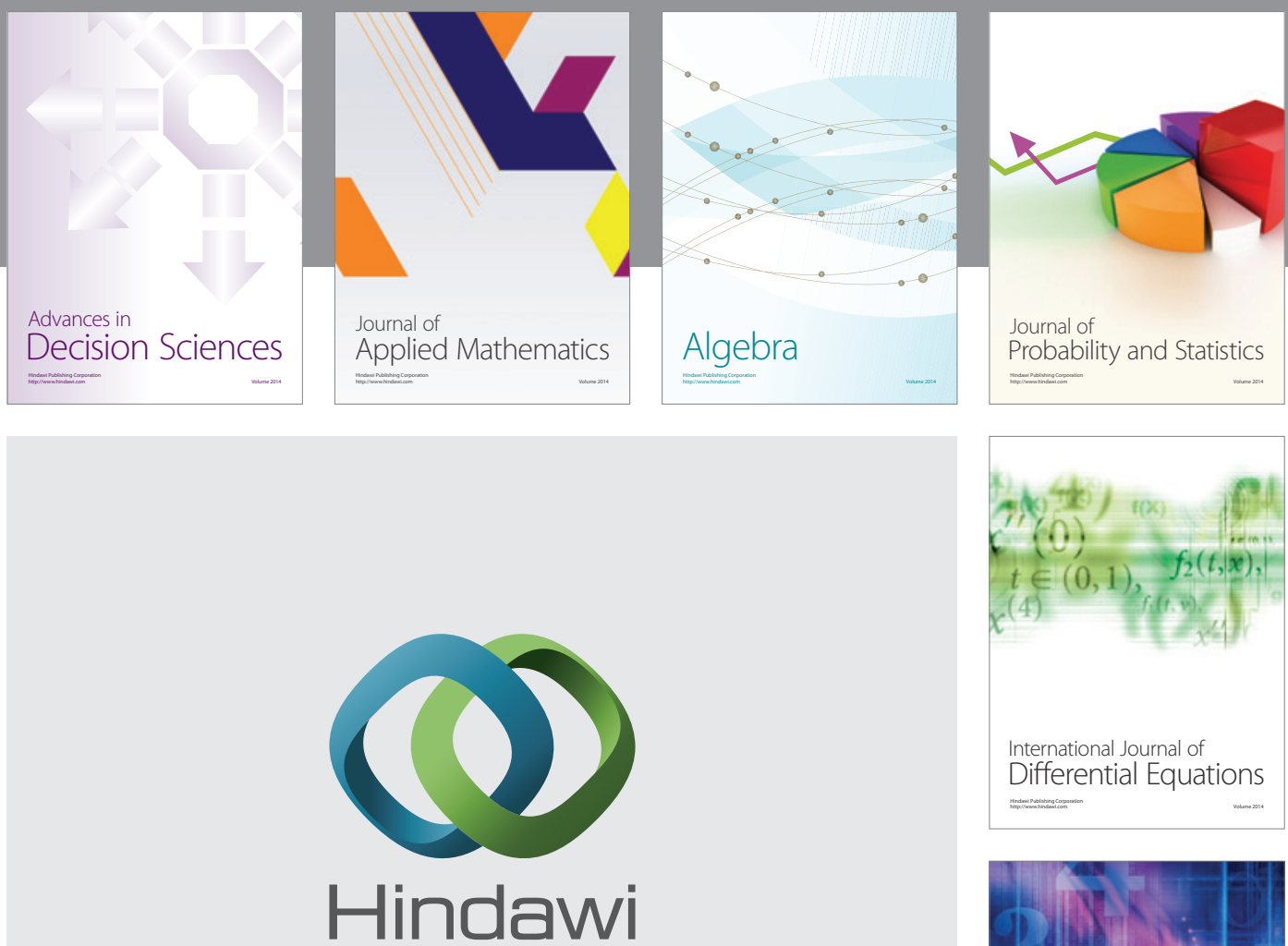

Submit your manuscripts at http://www.hindawi.com
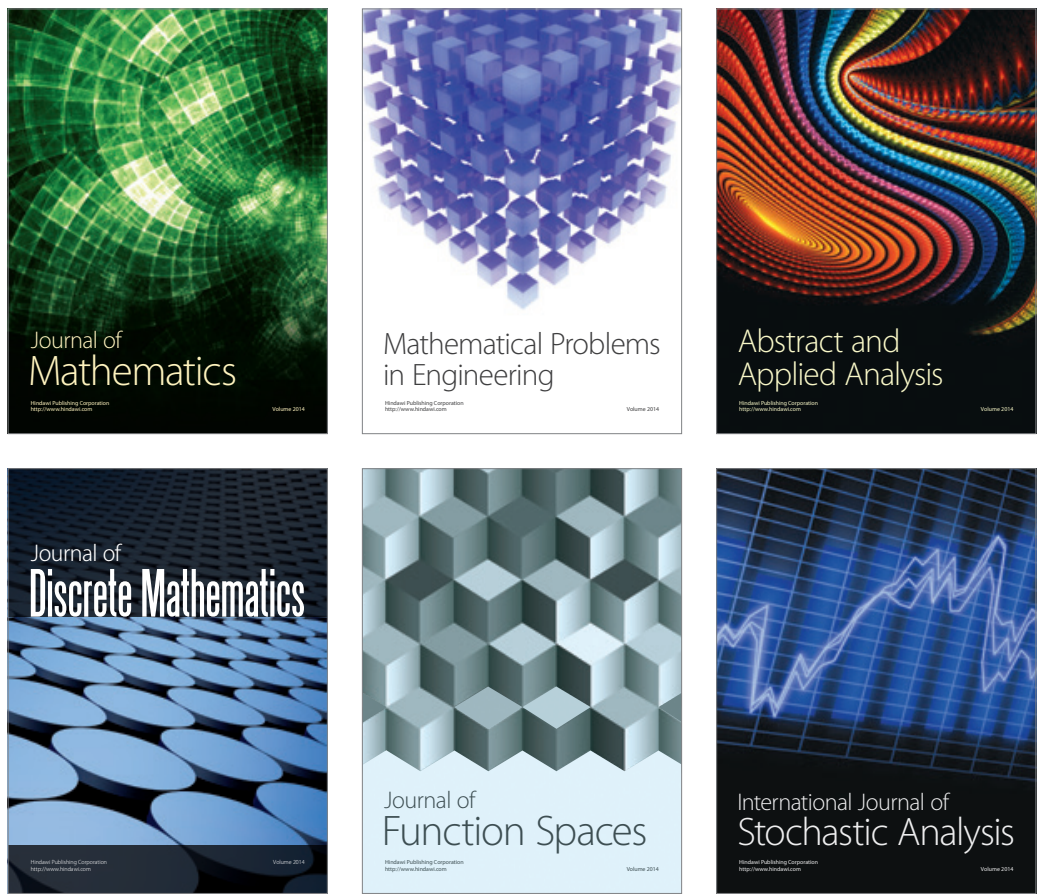

Journal of

Function Spaces

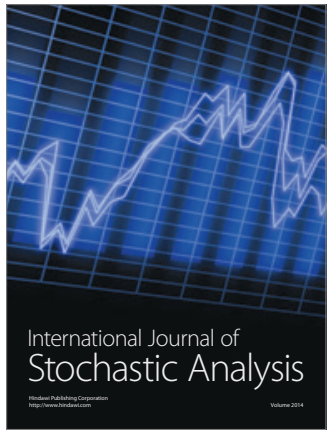

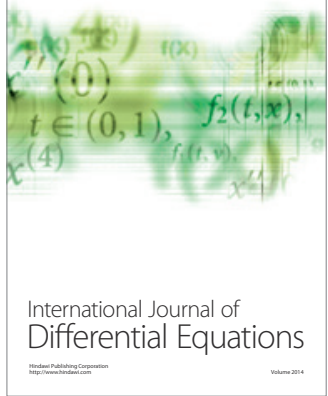
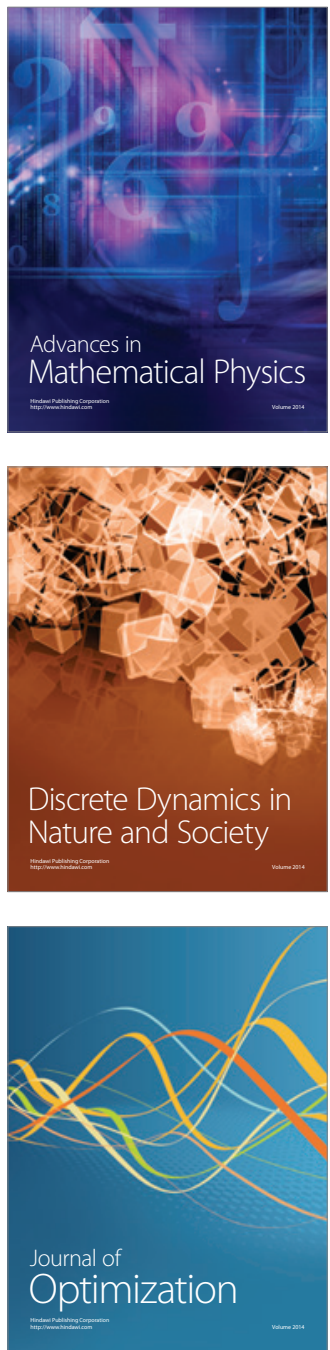